Material Properties

\title{
Evaluation of the Effect of Water Content on the Stress Optical Coefficient in PMMA
}

\author{
Orlando Delpino Gonzales ${ }^{\text {a }}$, Austin Nicassio ${ }^{\text {a }}$, Veronica Eliasson ${ }^{\text {a,* }}$ \\ ${ }^{a}$ Department of Aerospace and Mechanical Engineering, University of \\ Southern California, \\ 854 Downey Way, Los Angeles, CA, 90089
}

\begin{abstract}
A series of experiments was completed to study the effect of water content on the stress optical coefficient of PMMA samples under uniaxial tension. The stress optical coefficient is an inherent material property that is utilized in interferometric techniques, classical photoelasticity and the method of caustics to obtain deformation measurements and fracture values. The stress optical coefficient depends on the variation of the mechanical properties and the refractive index of the polymer, both of which are affected by water uptake. The main objective of this study was to quantify the variation of the stress optical coefficient with water uptake, so that it can be utilized in the analysis of visualization techniques and applied to deformation and fracture studies in humid or liquid environments. Experiments were performed at two different strain rates, $5 \times 10^{-4}-5 \times 10^{-3} \mathrm{~s}^{-1}$, on both dry and pre-conditioned samples. The preconditioned samples were submerged in distilled water for either 8 or 40 days, allowing samples to reach water contents of 0.8 wt $\%$ and 1.4 wt \%, respectively. Measurements were completed utilizing a Fizeau interferometer and high-speed photography. Results showed a variation on the stress optical coefficient of up to $11 \%$ for a water content of $0.8 \mathrm{wt} \%$ and a strain rate of $5 \times 10^{-4} \mathrm{~s}^{-1}$. This variation was no longer observed when the strain rate was increased by an order of magnitude.
\end{abstract}


Keywords: PMMA, Stress Optical Coefficient, Interferometry, Water Content

*Corresponding author.

Email address: eliasson@usc.edu (Veronica Eliasson)

\section{Introduction}

The experimental determination of stresses within a body under tension using non-contact visualization techniques in transmission requires measurements of the variations of the optical paths along the principal stress directions. These optical path differences are related to the applied stresses through the stress optical coefficient (SOC). It is known that the SOC depends on the variation of mechanical properties and the refractive index of a polymer, both of which are known to be affected by water [1, 2]. It is relevant to study these properties on poly(methyl methacrylate) (PMMA) because the applications for which this material can be utilized are principally based on its transparency and durability, making it a good candidate for use as a component in aerospace and naval structures (e.g. windows, lenses, canopy, cover, etc.). As a result, these applications often require the material to be exposed to an outdoor environment where it will be subjected to environmental effects. Among these effects, humidity or water exposure are conditions that cause aging in the material and are known to gradually degrade its useful properties.

The variation of the mechanical properties of PMMA due to water sorption has been investigated previously for different loading conditions, temperatures, geometries and compositions. These studies include deformation, fracture strength and crack propagation [1, 3-10]. For each of these studies, the maximum water content level reached at saturation is less than $2 \mathrm{wt} \%$ because PMMA and water do not have high chemical compatibility. It is 
well understood that the process of water sorption in PMMA follow Fick's diffusion law [11].

Shen et al. [1] performed experiments on samples subjected to accelerated aging by conditioning test specimens in water for 22 days at $60^{\circ} \mathrm{C}$. It was found that in PMMA the ductility initially increases with increasing water content but then reduces at higher concentrations above $1.1 \mathrm{wt} \%$, such that it appears that the brittleness increases for water contents higher than $1.1 \mathrm{wt} \%$. This phenomenon was attributed to the change in the mechanism by which water is accommodated in the PMMA microstructure as water concentration increases. It was suggested that at water concentrations above $1.1 \mathrm{wt} \%$, the behavior changes as water molecules begin to cluster and act more as filler particles than as a plasticizer. Thus, water acts predominantly as a mild plasticizer for PMMA at water contents of about $1.1 \mathrm{wt} \%$, but at higher water contents clustering occurs, which yields significant changes in the deformational response and a drop in tensile strength [1].

Arnold [11] completed quasi-static tensile tests at a strain rate of $1 \times 10^{-4} \mathrm{~s}^{-1}$ on PMMA samples pre-conditioned in water and other liquids for periods of time ranging from 1 minute to 14 days. Results showed that ductility increases with immersion time. It must be noted that all of their samples exhibited a water content lower than 1 wt $\%$ after conditioning, so the findings shown by Shen et al. [1] were not observed. Nevertheless, these results supported the concept that plasticization occurs when PMMA samples contain water concentrations lower than $1 \mathrm{wt} \%$.

Hamouda [5] studied the tensile deformation and fracture of PMMA on samples that were exposed to different humidity levels and immersed in water to achieve $0.8 \mathrm{wt} \%$ of water uptake. The experiments were made at a loading rate of $1 \mathrm{~mm} / \mathrm{min}$. Properties such as crazing, tensile fracture stress, 
strain-to-fracture and fracture surface morphology were investigated. It appears that this study was an extension of the work done by Shen et al. [1] since more cases under similar loading conditions were analyzed. The transition from brittle to ductile mode was clearly observed as the water content increased from 0 to $0.8 \mathrm{wt} \%$, which indicated that the material toughness increases with water content up to a concentration of $0.8 \mathrm{wt} \%$. In general, in agreement with Shen et al. [1], it was concluded that ductility increases with water content up to $0.8 \mathrm{wt} \%$. Furthermore, the tensile strength for PMMA reduces with increasing water content.

Ishiyama and Higo [3] performed tensile tests for three different humidity conditions at three different strain rates $\left(1 \times 10^{-3} \mathrm{~s}^{-1}, 1 \times 10^{-4} \mathrm{~s}^{-1}\right.$, $1 \times 10^{-5} \mathrm{~s}^{-1}$ ) in an effort to study the effect of humidity levels on the Young's modulus of PMMA samples exposed to their respective environments for 60 days. Results showed that strain rate and water content affected the Young's modulus of PMMA, such that it decreases with increasing water content.

All of the previously mentioned studies describe changes in the mechanical behavior of PMMA when subjected to quasi-static testing with varying amounts of sorbed water. Thus, it is apparent that the mechanical response of PMMA after water exposure is well understood for strain rates of $1 \times 10^{-5} \mathrm{~s}^{-1}-1 \times 10^{-3} \mathrm{~s}^{-1}$. On the other hand, the effect of water sorption on the optical properties of PMMA is not yet clear. To the best of the authors' knowledge, the most relevant research on this subject was done by Abo-El Ezz et al. [2], who studied the variation of the optical constants of PMMA when immersed in a liquid environment. However, this study did not include the effects of water sorption after pre-conditioning the samples for 
certain periods of time, but rather the immediate effect of the surrounding liquid during the experiments.

In order to study the effect of water sorption on the SOC, it is necessary to apply a technique capable of relating the stress applied to the optical path variation. One of the methods that has been utilized to study the SOC is called a Fizeau interferometer. This technique was introduced by Theocaris and Gdoutos [12] as an experimental method for solving plane-stress elastic problems. A Fizeau interferometer is based on individual measurements of the absolute retardation of monochromatic light. This method uses the specimen as the interferometer, and the interference fringes obtained are of the Haidinger type. One of the main advantages of this technique is that the samples do not need to be optically flat provided that the deviations from flatness are not abrupt. Additionally, it is one of the most straightforward methods based on measurements of the absolute variations of the optical paths for the solution of elasticity problems because it does not use any external elements for creating interference, and it has an uncomplicated optical setup. The main disadvantage of the method is that the principal stress directions must be known beforehand, but this is easily determined in uniaxial tensile tests. Raftopoulos et al. [13] applied this technique to study the static and dynamic SOC of dry PMMA specimens. The authors provided an in-depth description of their experimental setup, which was utilized to build the experimental setup used to obtain the results presented in this manuscript.

The motivation for this study resides in the need to obtain precise values of the SOC under different environmental conditions. These values could be used for studies of deformation and fracture in various visualization techniques such as interferometry, classical photoelasticity and the method 
of caustics. For this reason, the SOC of PMMA samples with different water contents was measured using a Fizeau interferometer during tensile testing. Additionally, the SOC behavior of PMMA was separated into optical and mechanical properties, as also done in [12], to independently study and compare each response and understand the effect that sorbed water has on the optical and mechanical contributors of the SOC of PMMA.

\section{Method Description}

A Fizeau interferometer was employed to quantify the effect of water on the SOC of PMMA samples. A monochromatic and coherent light source with a wavelength of $\lambda=632 \mathrm{~nm}$ was used, similar to that utilized in [13]. The light is normally incident on a thin plate under generalized plane-stress conditions to obtain a reflection from the front and rear faces of the sample.

The absolute variations of the two reflections from the front and rear faces of the plate results in a full field map containing information about the thickness and refractive index of the samples in their unloaded state [12].

The interference created from the reflection of each surface creates welldefined fringes. Once a load is applied on the specimen, a displacement of the interference fringes along the field is created due to the change in thickness of the sample. By counting the number of fringes passing across each point, the SOC can be calculated. The reason this technique is applied is, not only because it is straightforward, but it was also shown that a Fizeau interferometer is significantly more sensitive and accurate than any other interferometric method using an external interferometer or classical photoelasticity to perform coefficient measurements [12]. The theoretical analysis of stress-optical relationships that result in the equations used for the Fizeau interferometer are not described here, since the theoretical derivation 
has appeared in previous work, see for example references [12-14]. Only the final formulation of the previous analysis is shown. The assumptions made to arrive at these equations are that PMMA is an optically isotropic material, also called optically inert, which means that its optical properties are the same in all directions, and that the fringe count will be the same in both principal stress directions $[12,13]$.

The equation used to calculate the SOC in transmission, $C_{t}$, is given by

$$
C_{t}=\frac{\alpha_{t}+\beta_{t}}{2}
$$

where $\alpha_{t}$ and $\beta_{t}$ are the stress optical coefficients in transmission for each principal direction 1 and 2, respectively. Equation 1 is derived from the Maxwell-Neumann stress-optical law. This equation accounts for the change in thickness of the material and how it affects the interaction of light rays reflected from the front and rear face of the sample.

Additionally, mechanical properties such as Young's modulus, $E$, and Poisson's ratio, $v$, are included in the analysis. The equations used to relate the number of fringes counted along the two principal stress directions, $N_{l}$ and $N_{2}$, during loading and the stress optical constants describing the interference caused by reflected rays, $\alpha^{*}$ and $\beta^{*}$, for uniaxial tension test $\left(\sigma_{2}=0\right)$ are

$$
\begin{aligned}
& \alpha^{*}=\frac{N_{1}}{2 b \sigma} \\
& \beta^{*}=\frac{N_{2}}{2 b \sigma}
\end{aligned}
$$

where $b$ is the thickness of the samples and $\sigma$ is the load applied.

To relate the coefficients $\alpha_{t}$ and $\beta_{t}$ and the constants $\alpha^{*}$ and $\beta^{*}$, the relations $\alpha_{t}=-\lambda \alpha^{*}+v / E$ and $\beta_{t}=-\lambda \beta^{*}+v / E$ can be utilized. Since PMMA is 
an optically isotropic material and presents very weak birefringence, it has been previously proven that the fringe count in the principal directions are equal, i.e. $N_{1}=N_{2}$, which means that $\alpha^{*}=\beta^{*}$ [13]. By combining Equation 1 and 2 , the SOC can be explicitly expressed by

$$
C_{t}=-\lambda \frac{N_{1}}{2 b \sigma}+\frac{v}{E}
$$

Equation 4 shows a direct contribution from both mechanical and optical properties. This will be utilized to further analyze the contribution of water content on the variation of the optical or mechanical properties of the material.

\section{Material and Conditioning}

Commercially available PMMA (from Emco Industrial Plastics, Inc) was used for these experiments. All samples met the ASTM D638-10 (Type II) standards with dimensions of $3.125 \mathrm{~mm}$ thickness, $183 \mathrm{~mm}$ overall length and $19 \mathrm{~mm}$ overall width, as shown in Figure 1.

\section{(Figure 1 Location)}

Experiments were performed using samples that were submerged in distilled water, as well as dry samples for comparison purposes. It is known that water content increases with time of exposure [10]. For this reason, samples were submerged in distilled water for 8 and 40 days. These exposure time durations were chosen such that water content was lower than $1.1 \mathrm{wt} \%$ for the samples exposed to water for 8 days and higher than $1.1 \mathrm{wt}$ $\%$ for samples immersed in water for 40 days. Prior to placing the samples in their respective environments, they were dried in an air oven at $70^{\circ} \mathrm{C}$ for 24 hours, in compliance with the method described in [3]. The weight change in the samples during their exposure time was periodically monitored using a balance (ML4002E/03, Mettler Toledo), with a 
readability of $0.01 \mathrm{~g}$, to obtain the water content as a function of time. Water absorption percentage was calculated using Equation 5

$$
M=\frac{W_{f}-W_{i}}{W_{i}} \times 100
$$

where $M($ wt $\%)$ is the water content, $W_{i}$ is the weight of a sample before immersion, and $W_{f}$ is the weight of the sample containing sorbed water. Figure 2 shows the weight measurements as a function of time. It was observed that the water content of each sample increased with time, but the rate of absorption decreased with time, in agreement with [4]. As can be seen in Figure 2, there was a weight increment of 0.69 wt \% due to water sorption after the first five days of conditioning. This sorption rate decreased with time such that in the last ten days of conditioning only a 0.17 wt $\%$ weight increment was observed. The resultant water content for the samples exposed to water for 8 and 40 days was 0.8 wt $\%$ and 1.4 wt $\%$, respectively.

(Figure 2 Location)

\section{Experimental Procedure}

A series of experiments under uniaxial tension were completed using a 5567 Instron machine with a $5 \mathrm{kN}$ load cell. Experiments for each conditioning set were performed at strain rates of $5 \times 10^{-4} \mathrm{~s}^{-1}$ and $5 \times 10^{-3} \mathrm{~s}^{-1}$ with loads increasing from zero up to fracture at a constant temperature of $25^{\circ} \mathrm{C}$. A small pre-load of $40 \mathrm{~N}$ was applied to all samples prior to each experiment to ensure alignment. The experimental setup is shown in Figure 3. A He-Ne laser with a wavelength of $\lambda=632 \mathrm{~nm}$ and a $10 \mathrm{~mm}$ beam diameter, generated by using a $15 x$ beam expander, was used to impinge light onto the sample. A Phantom V711 high-speed camera was used to record the fringes as the samples were loaded, allowing full-field 
visualization, which yielded multiple points for analysis. Figure 4 shows a representative image of the appearance of the well-defined fringes captured by the camera. The image of the specimen located next to the fringes in Figure 4 shows the field of view observed during the experiments. The highspeed camera was set to a resolution of 750x300 pixels and an exposure time of $30 \mu \mathrm{s}$. A $200 \mathrm{~mm}$ lens (AF Micro-Nikkor, f/4D) was used, resulting in a scale of $70 \mu \mathrm{m} /$ pixel. The high-speed camera was set to record at 24 and 300 fps for the experiments performed at strain rates of $5 \times 10^{-4} \mathrm{~s}^{-1}$ and $5 \times 10^{-3} \mathrm{~s}^{-1}$, respectively. These frame rates were carefully selected to minimize measurement errors by recording and averaging results from 8-10 frames per experiment.

(Figure 3 Location)

(Figure 4 Location)

Measurements such as stress and strain were obtained directly from the load cell in the Instron machine in addition to a clip-on extensometer added onto each sample. The sampling rate of the Instron machine was 0.1 seconds, which yielded an average of 100 and 1000 data points for the samples loaded at the strain rates of $5 \times 10^{-3} \mathrm{~s}^{-1}$ and $5 \times 10^{-4} \mathrm{~s}^{-1}$, respectively. By using the stress measured by the load cell and the fringe count obtained from the recorded images, the SOC was calculated as the load increased. The correlation between the images recorded and the stress/strain data was performed by shifting the time data so that the time instant when fracture occurred was set as a common point in time.

The post-processing procedure was completed using MATLAB to obtain the elastic modulus and to count the pixel intensity at multiple sample locations. To do this, two points within the field of view of the sample were selected, which represented the endpoints of a line along which the 
intensity of every pixel was measured as the load was increased. This yielded a time history of the intensity of each pixel. In this work, data from several pixels was obtained for analysis, contrary to the method employed by Raftopoulos et al. [13] who only measured a single point. Figure 5 shows a digitized image of the fringe count obtained for the intensity of a single pixel as a function of time. To facilitate and automate the fringe count, a zero-phase digital filter was used to smooth the data. Each peak was considered a fringe, thus, once the data was smoothed out, fringe count was easily performed. Once the fringes (or peaks) were computed, the fringe count was grouped in intervals of applied stress level. Lastly, Equation 4 was used to calculate SOC values as the load was increased.

\section{(Figure 5 Location)}

\section{Results and Discussion}

As previously mentioned, the values that represent the mechanical response of the material were directly measured from the load cell and clipon extensometer used during the experiments, and the values for the optical response were measured with the Fizeau interferometer and high-speed photography. The results from both sources of measurements were compared to confirm their agreement; thus, the fringe count, which represents the change in thickness of the material, was normalized by the maximum number of counted fringes and compared with the normalized strain obtained from the clip-on extensometer. These two terms are proportional to the change in thickness of the material as it is loaded. Figure 6 shows an example of the results when they are compared. Their agreement confirms that the fringe count is a measurement that accurately represents the sample's behavior. This type of comparison was performed during the 
analysis of every sample to ensure the fidelity of the measurements obtained from the Fizeau interferometer.

\section{(Figure 6 Location)}

The variation of the mechanical response of PMMA was measured by investigating the effect of water content on its elastic modulus. Figure 7 shows the variation of the elastic modulus measured at two different strain rates as a function of water content. The elastic modulus decreased with increasing water content for both strain rates, and increased with increasing strain rate. The effect of strain rate on PMMA is well understood as it is known that it is highly rate dependent, such that it experiences an incremental increase on the elastic modulus as strain rate increases. This can be attributed to the fact that, as the strain rate increases, the molecular mobility of the polymer chains will be decreased, thus making the chains stiffer, and a stiffer material has a higher elastic modulus $[15,16]$. Additionally, the effect of water content on the mechanical response of PMMA agrees with the findings by $[3,17]$. The effect of water is known to act as a mild plasticizer, and thus results in a decrease in the elastic modulus. In this study, the analysis of the mechanical response of PMMA is focused on the variation of its elastic modulus because, as seen in Equation 4, it is a critical term for the calculation of the SOC. Another mechanical property involved in the analysis of the SOC is Poisson's ratio. However, it was reported by Theocaris [14] that the Poisson's ratio does not have a significant variation in PMMA. For this reason, in this study, Poisson's ratio was assumed to remain constant, $v=0.36$.

(Figure 7 Location)

Figures 8(a) and 8(b) show the variation of the SOC for the three different conditioning scenarios at both strain rates studied. Each of these 
curves feature results for at least 5 samples per case. Note that only a few points from the entire data set are shown for ease of reporting the results. However, the trends and values are well represented in these plots. The values calculated for the SOC immediately prior to fracture agree with the results reported in the literature by Katsamanis et al. [18], who reported values of $c_{t}=1.21 \times 10^{-10} \mathrm{~m}^{2} / \mathrm{N}$, and Theocaris [14] who reported values of $c_{t}=1.1 \times 10^{-10}$ to $1.7 \times 10^{-10} \mathrm{~m}^{2} / \mathrm{N}$. It has also been reported that the SOC is strain rate dependent. In this study, a reduction of the SOC of $10+/-4 \%$ was observed as the strain rate was increased by an order of magnitude. This behavior has been observed in comparisons between dynamic and static loading for which reductions of up to $40 \%$ in the SOC values have been reported $[13,18]$.

(Figure 8(a) Location)

(Figure 8(b) Location)

Overall, the variation of the SOC due to water content is lower than 11 $+/-7 \%$. For both strain rates applied, the behavior of the SOC in samples pre-conditioned in water is similar regardless of the duration of time that they were immersed in water. To further understand the effect of water content on the SOC, the approach adopted was to study the contribution from the optical and mechanical properties of the material by independently analyzing the response from the first and second terms in Equation 4, respectively. Figure 9(a) and 9(b) show the contributions from each term $\left(\lambda \alpha^{*}\right.$ and $\left.v / E\right)$ as the load increased for samples subjected to strain rates of $5 \times 10^{-3} \mathrm{~s}^{-1}$ and $5 \times 10^{-4} \mathrm{~s}^{-1}$, respectively.

The comparison analysis was performed by studying the variation of the results of the samples pre-conditioned in a water environment against the results obtained for the dry samples. From Figures 9(a) and 9(b), it is apparent 
that, for all cases, the dominant contribution is the term representing the optical properties. Figure 9(a) shows that a similar effect was experienced by both mechanical $(v / E)$ and optical $\left(\lambda \alpha^{*}\right)$ contributions for samples that were immersed in water for the same time duration. Furthermore, the magnitude of variation of both mechanical and optical contributions due to water content was higher for the samples with a water content of $1.4 \mathrm{wt} \%$. On the other hand, Figure 9(b) shows that the variation in the optical properties was similar for samples preconditioned in a water environment, yet their mechanical properties showed dissimilar behavior. The samples with a water content of $1.4 \mathrm{wt} \%$ experienced significant variation in their mechanical response compared to the samples with a water content of $0.8 \mathrm{wt} \%$. A change in the response of the optical properties seems to occur as water content is increased in the samples tested at a strain rate of $5 \times 10^{-4} \mathrm{~s}^{-1}$. However, this behavior was not observed for experiments performed at a strain rate of $5 \times 10^{-3} \mathrm{~s}^{-1}$. Further studies must be completed to understand the mechanism causing this response.

(Figure 9(a) Location)

(Figure 9(b) Location)

To summarize these results, Table 1 shows the variation experienced by both contributing terms for each of the cases studied. It must be noted that the values shown represent an average variation from the dry samples for the duration of the experiment. Figures 9(a) and 9(b) show a complete trend and distribution of the results. 
Table 1: Average variation from dry conditions of optical $\left(\lambda \alpha^{*}\right)$ and mechanical $(v / E)$ contributions due to water sorption

\begin{tabular}{|l|l|l|l|l|}
\hline Case & $\dot{\varepsilon}$ & $v / E$ & $\lambda \alpha^{*}$ & $C_{t}$ \\
\hline $0.8 \mathrm{wt} \%$ & $5 \times 10^{-3} \mathrm{~s}^{-1}$ & $4+/-4 \%$ & $1+/-3 \%$ & $3+/-7 \%$ \\
\hline $0.8 \mathrm{wt} \%$ & $5 \times 10^{-4} \mathrm{~s}^{-1}$ & $3+/-5 \%$ & $14+/-2 \%$ & $11+/-7 \%$ \\
\hline $1.4 \mathrm{wt} \%$ & $5 \times 10^{-3} \mathrm{~s}^{-1}$ & $21+/-2 \%$ & $18+/-3 \%$ & $3+/-5 \%$ \\
\hline $1.4 \mathrm{wt} \%$ & $5 \times 10^{-4} \mathrm{~s}^{-1}$ & $20+/-1 \%$ & $13+/-3 \%$ & $7+/-4 \%$ \\
\hline
\end{tabular}

Table 1 shows that water content had the most influence, at both strain rates applied, on the mechanical properties in the cases where the samples had a water content of $1.4 \mathrm{wt} \%$, reaching values of up to $20 \%$ variation. The change in the optical properties was slightly lower, reaching values of up to $18 \%$ variation. The last column of Table 1 shows the variation of the SOC as a result of water content. Due to the nature of Equation 4, the condition that determines the overall effect of water content on the SOC is the difference between the contributions of the mechanical properties over the optical ones. As can be seen, the average values of the SOC are a good representation of what is displayed in Figure 8. A small effect of water content on the SOC for samples subjected to a strain rate of $5 \times 10^{-4} \mathrm{~s}^{-1}$ is observed, and this effect was reduced with increasing strain rate. Hence, there was no significant difference in the SOC between dry samples and pre-conditioned samples for the strain rate of $5 \times 10^{-3} \mathrm{~s}^{-1}$.

\section{Conclusions}

The effect of water content on the SOC of PMMA when subjected to two different strain rates under quasi-static loading was investigated. The 
SOC was measured by using a Fizeau interferometer and high-speed photography. The results can be concluded as follows:

- Water content had an effect on the mechanical contribution, $v / E$, of the SOC by causing a variation of up to $20 \%$ from dry conditions. The effect of water on the optical contributions, $\lambda \alpha^{*}$, of the SOC was marginally lower, reaching values of up to $18 \%$ variation from dry conditions.

- Since the effect of water content on the optical and mechanical contributors was similar, this yielded a variation in the SOC of up to $11 \%$ for samples subjected to a strain rate of $5 \times 10^{-4} \mathrm{~s}^{-1}$. This effect was reduced with increasing strain rate, such that there was no significant difference in the values of the SOC between dry and preconditioned samples for the experiments performed at the strain rate of $5 \times 10^{-3} \mathrm{~s}^{-1}$.

\section{Acknowledgements}

The authors gratefully acknowledge the support of the Office of Naval Research through Grant Number N000141310607 (Dr. Y.D.S. Rajapakse, Program Manager) and the National Science Foundation through Grant Number CMMI-1332840. Additionally, the authors would like to thank Professor S. Nutt from the USC Viterbi M.C. Gill Composites Center for allowing us to use his facilities and Mr. Y. Zhang for his assistance during experiments utilizing the Instron machine.

\section{References}

[1] J. Shen, C. Chen, J. Sauer, Effects of sorbed water on properties of low and high molecular weight PMMA: 1. Deformation and fracture behaviour, Polymer 26 (1985) 511-518. 
[2] A. E.-S. Abo-El Ezz, N. Takeda, K. Takahashi, Experimental determination of stress-optical constants of poly (methyl methacrylate) immersed in liquid environments, Journal of Physics D: Applied Physics 22 (5) (1989) 687.

[3] C. Ishiyama, Y. Higo, Effects of humidity on Young's modulus in poly(methyl methacrylate), Journal of Polymer Science Part B: 40 (2002) 460-465.

[4] Y. Bokoi, C. Ishiyama, M. Shimojo, Y. Shiraishi, Y. Higo, Effects of sorbed water on crack propagation in poly(methyl methacrylate) under static tensile stress, Journal of Materials Science 35 (2000) 5001-5011.

[5] A. Hamouda, The influence of humidity on the deformation and fracture behaviour of PMMA, Journal of Materials Processing Technology 124 (2002) 238-243.

[6] C. Ishiyama, T. Sakuma, M. Shimojo, Y. Higo, Effects of humidity on environmental stress cracking behavior in poly(methyl methacrylate), Journal of Polymer Science Part B: Polymer Physics 40 (2002) 1-9.

[7] C. Chen, J. Shen, J. Sauer, Effects of sorbed water on properties of low and high molecular weight PMMA: 11. Fatigue performance, Polymer 26 (1) (1985) 89-96.

[8] P. Burchill, Effect of absorbed water, temperature and strain rate on the yield strength of two methylmethacrylate-based plastics, Journal of Materials Science 24 (1989) 1936-1940.

[9] H. Wu, G. Ma, Y. Xia, Experimental study of tensile properties of PMMA at intermediate strain rate, Materials Letters 58 (29) (2004) $3681-3685$. 
[10] O. Delpino Gonzales, V. Eliasson, Influence of Water Uptake on Dynamic Fracture Behavior of Poly (Methyl Methacrylate), Experimental Mechanics, doi:10.1007/s11340-015-0030-4 (2015) 1-10.

[11] J. Arnold, The effects of diffusion on environmental stress crack initiation in PMMA, Journal of Materials Science 33 (21) (1998) 51935204.

[12] P. Theocaris, E. Gdoutos, An interferometric method for the direct evaluation of principal stresses in plane-stress fields, Journal of Physics D: Applied Physics 7 (1974) 472-482.

[13] D. Raftopoulos, D. Karapanos, P. Theocaris, Static and dynamic mechanical and optical behaviour of high polymers, Journal of Physics D: Applied Physics 9 (5) (1976) 869.

[14] P. Theocaris, Dependence of stress-optical coefficients on the mechanical and optical properties of polymers, The Journal of Strain Analysis for Engineering Design (4) (1973) 267-276.

[15] J. Richeton, S. Ahzi, K. Vecchio, F. Jiang, R. Adharapurapu, Influence of temperature and strain rate on the mechanical behavior of three amorphous polymers: characterization and modeling of the compressive yield stress, International Journal of Solids and Structures 43 (7) (2006) 2318-2335.

[16] W. Chen, F. Lu, M. Cheng, Tension and compression tests of two polymers under quasi-static and dynamic loading, Polymer Testing 21 (2) (2002) 113-121.

[17] L. Josserand, R. Schirrer, P. Davies, Influence of water on crack propagation in poly methyl methacrylate: craze stress and craze fibril lifetime, Journal of Materials Science 30 (1995) 1772-1780. 
[18] F. Katsamanis, D. Raftopoulos, P. Theocaris, Static and dynamic stress intensity factors by the method of transmitted caustics, Journal of Engineering Materials and Technology 99 (2) (1977) 105-109. 
Figure 1 caption

Drawing of tensile test sample. This shape corresponds to Type II ASTM D638-10 with a thickness of $3.125 \mathrm{~mm}$

Figure 2 caption

Weight increment due to water sorption on PMMA samples for an immersion time of up to 40 days

Figure 3 caption

Interferometer setup: (1) Phantom V711 high-speed camera, (2) PMMA sample, (3) $15 \mathrm{X}$ beam expander, (4) He-Ne laser with $\lambda=632 \mathrm{~nm}$

Figure 4 caption

Field of view and fringe appearance (Resolution: $70 \mu \mathrm{m} /$ pixel)

Figure 5 caption

Intensity plot showing fringe time history for a single pixel and its simultaneous loading history

Figure 6 caption

Comparison of response of extensometer and fringe count as a function of stress applied. Excellent agreement is obtained between the measurements

Figure 7 caption

Elastic modulus variation as a function of water content for two different strain rates $\left(5 \times 10^{-3} \mathrm{~s}^{-1}\right.$ and $\left.5 \times 10^{-4} \mathrm{~s}^{-1}\right)$

Figure 8 caption

Subcaption (a) $5 \times 10^{-3} \mathrm{~s}^{-1}$ 
Subcaption (b) $5 \times 10^{-4} \mathrm{~s}^{-1}$

Stress optical coefficient comparison for samples subjected to two different strain rates and with different water contents

Figure 9 caption

Subcaption (a) $5 \times 10^{-3} \mathrm{~s}^{-1}$

Subcaption (b) $5 \times 10^{-4} \mathrm{~s}^{-1}$

Optical and mechanical contributions to SOC for samples at two different strain rate 
$50 \mathrm{~mm}$

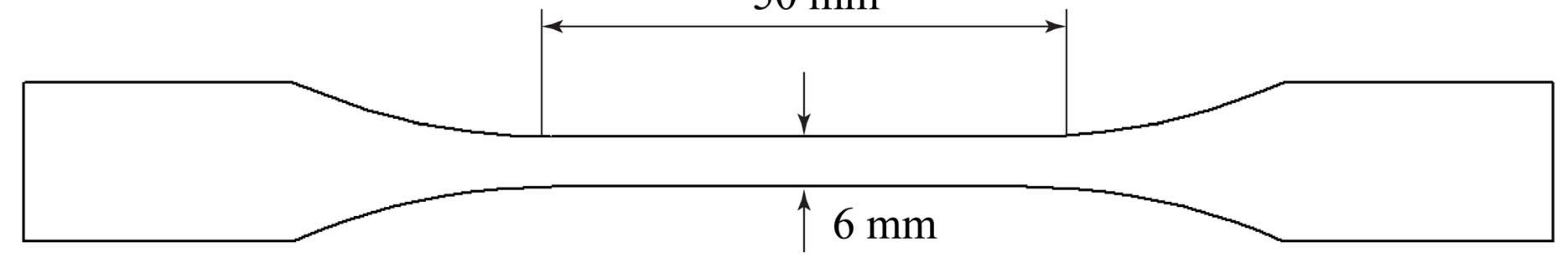




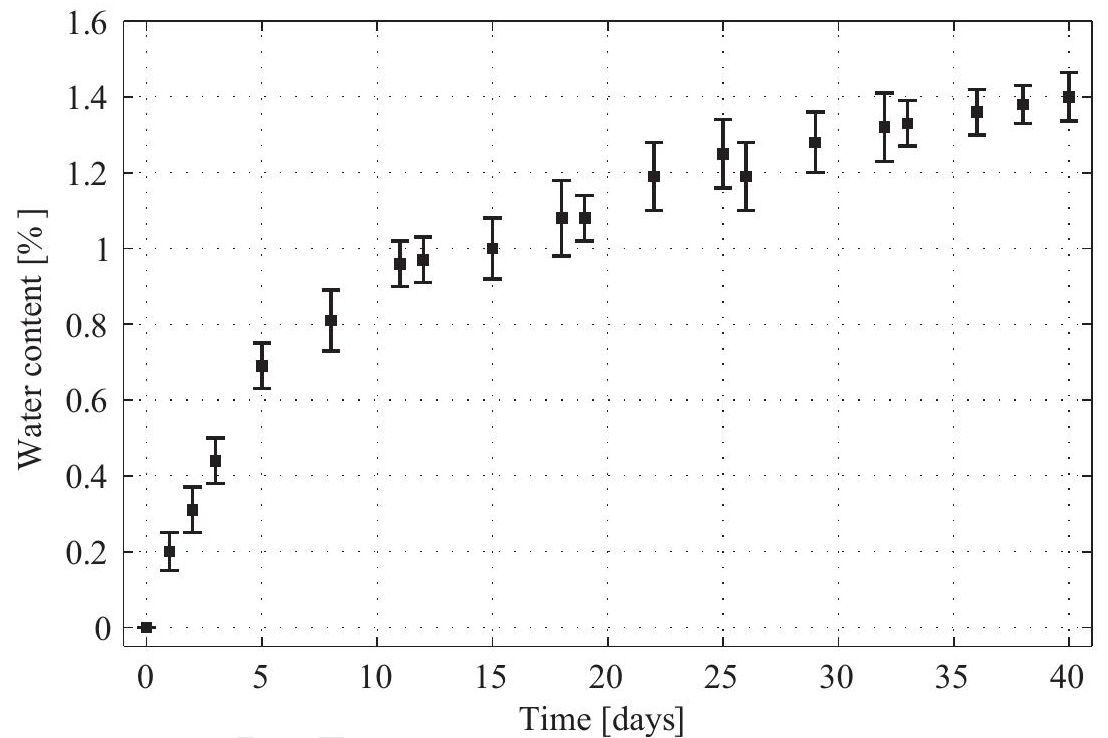




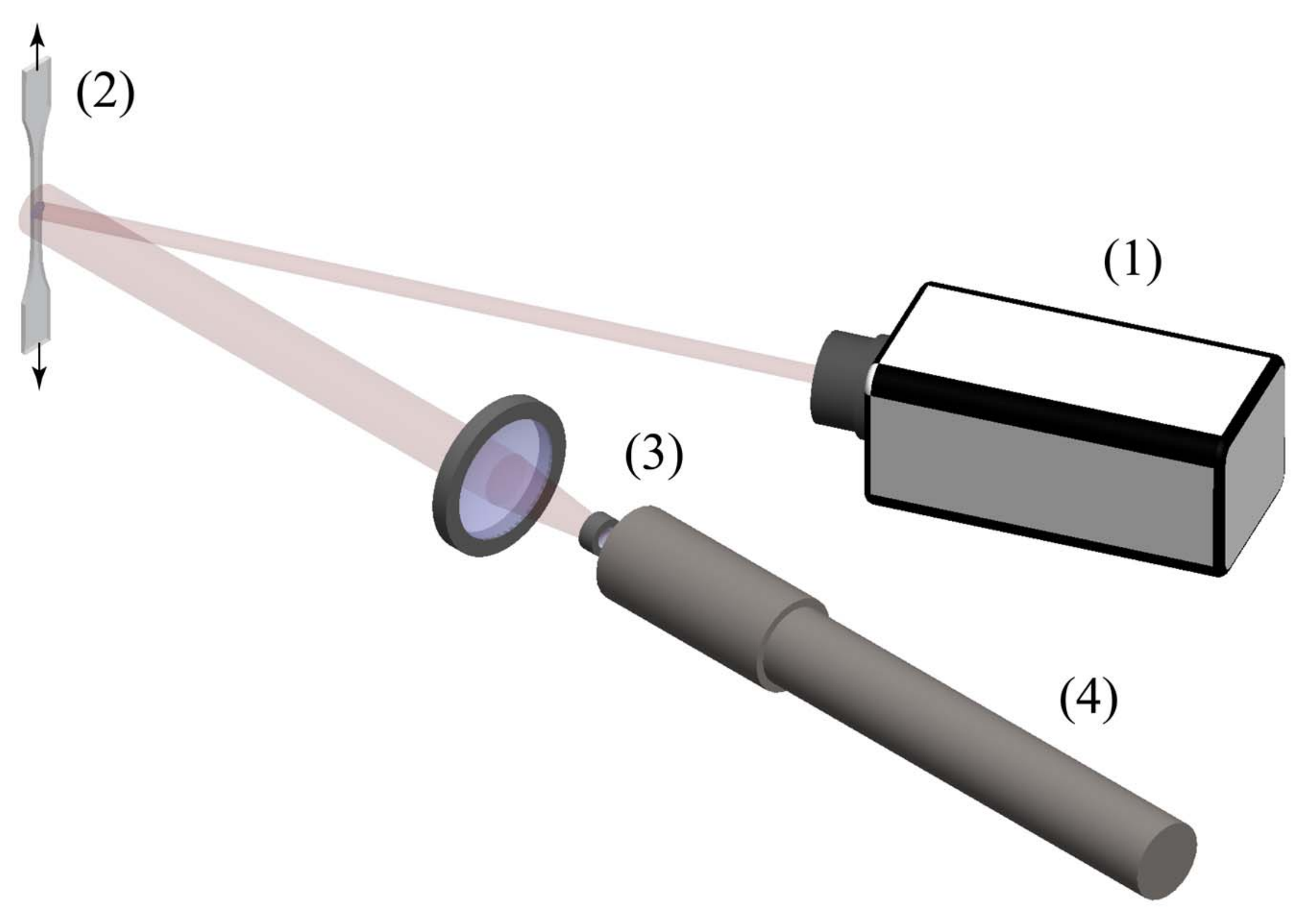




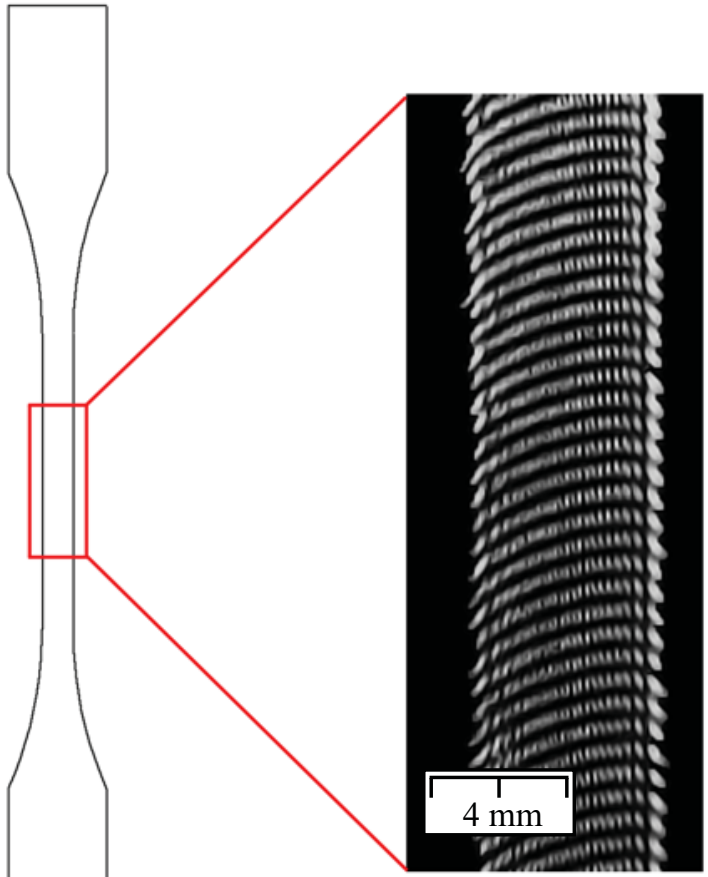




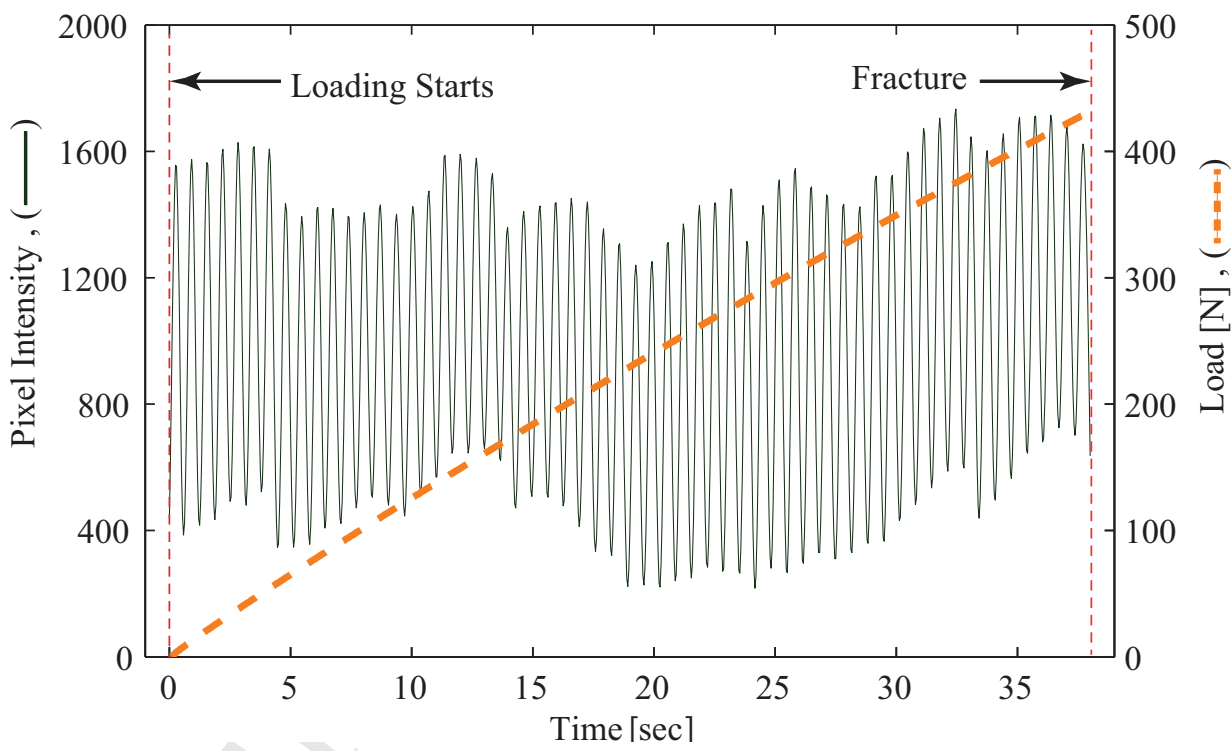




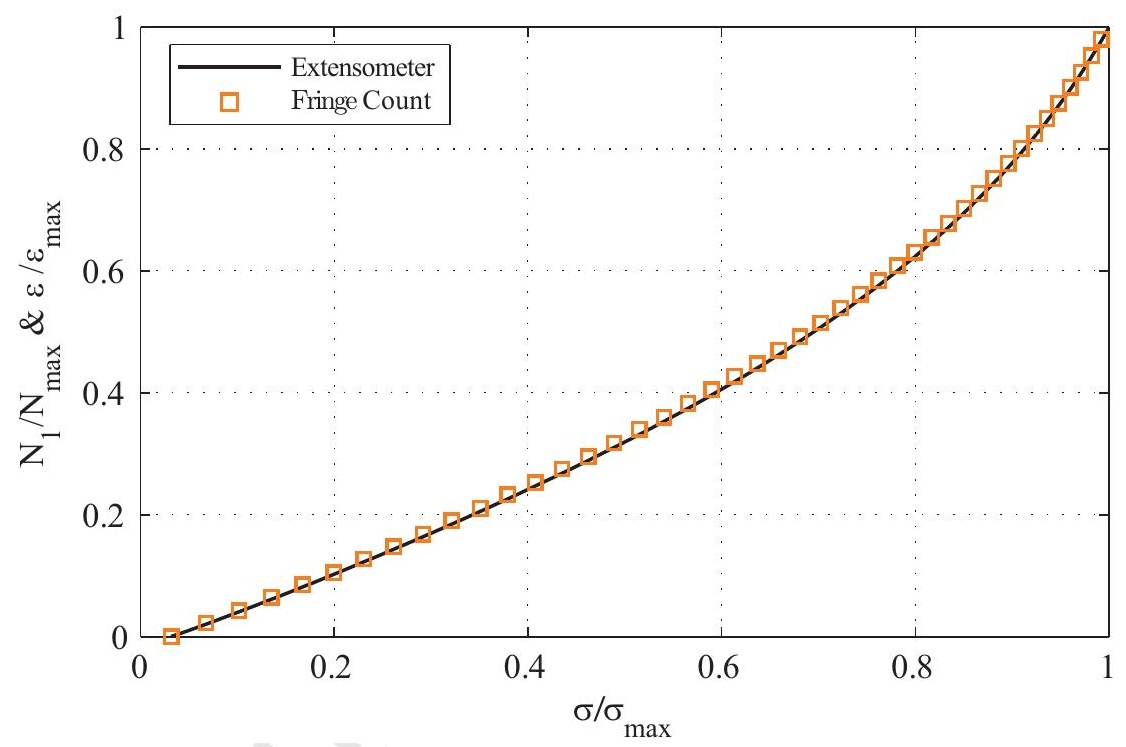




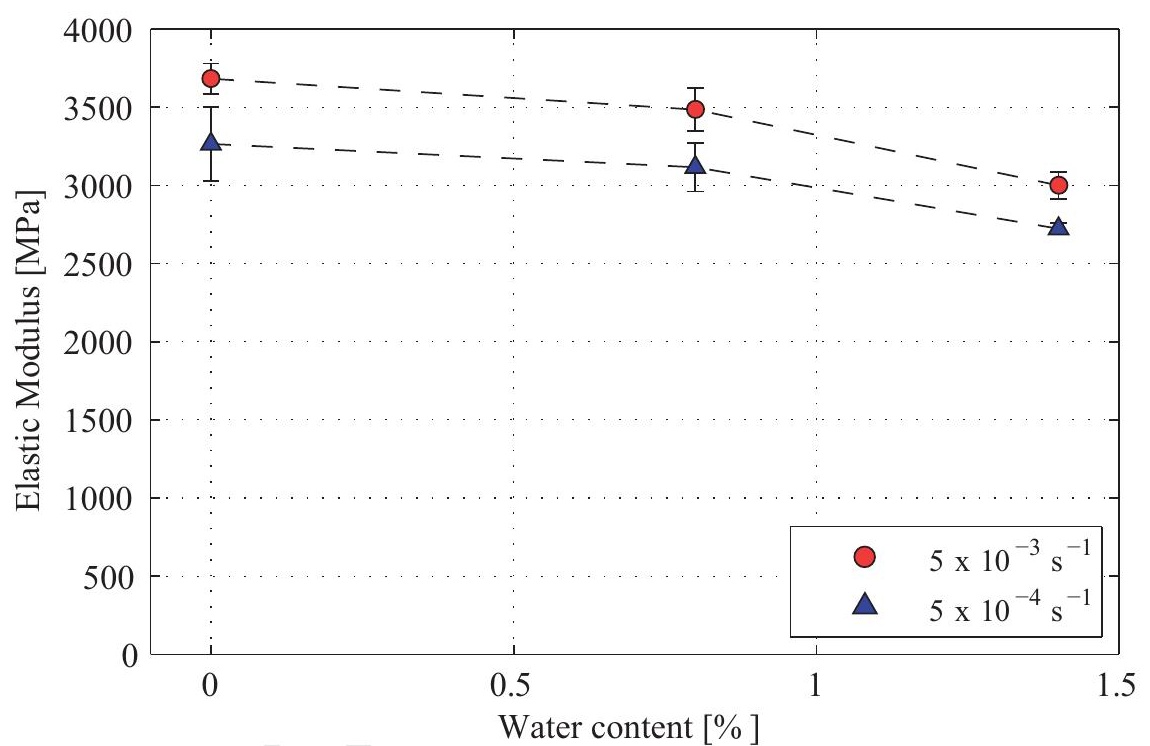




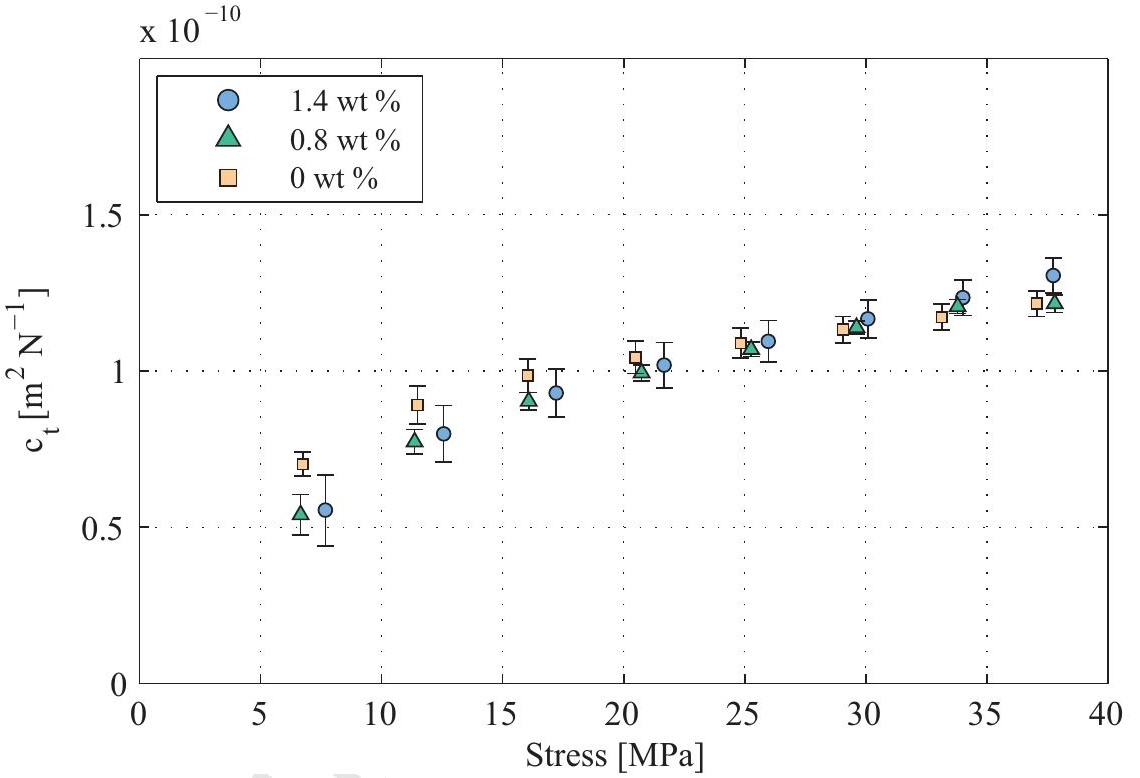




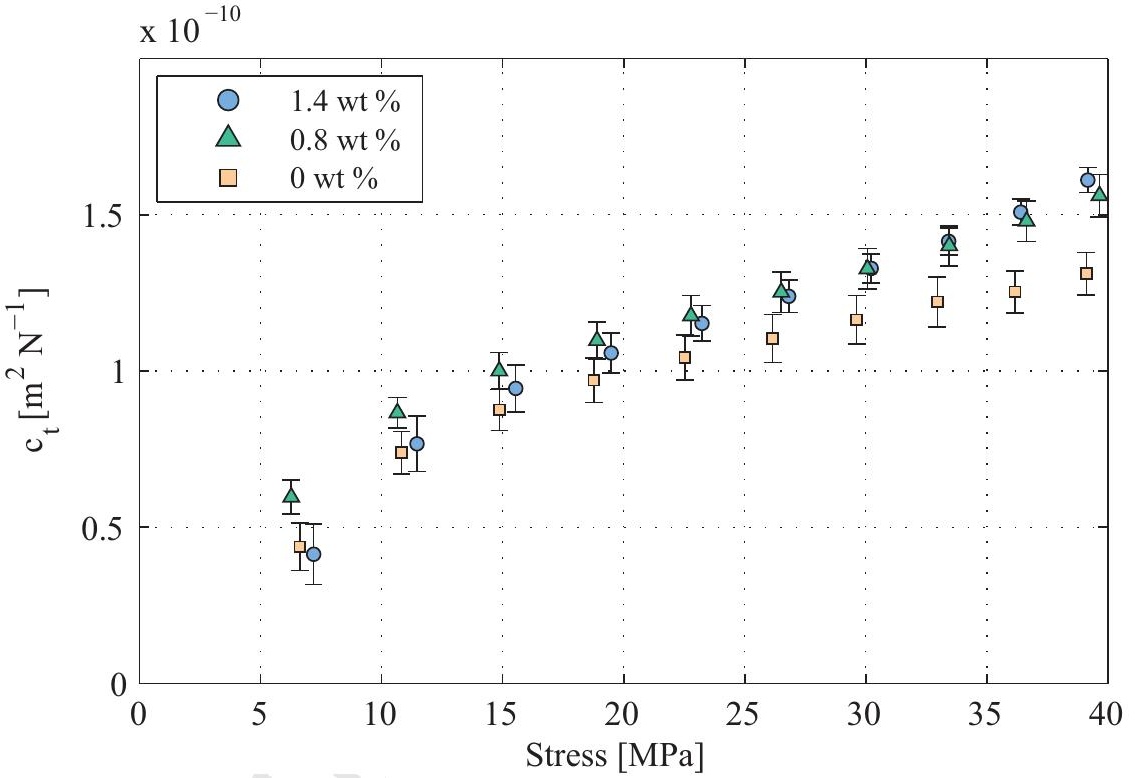




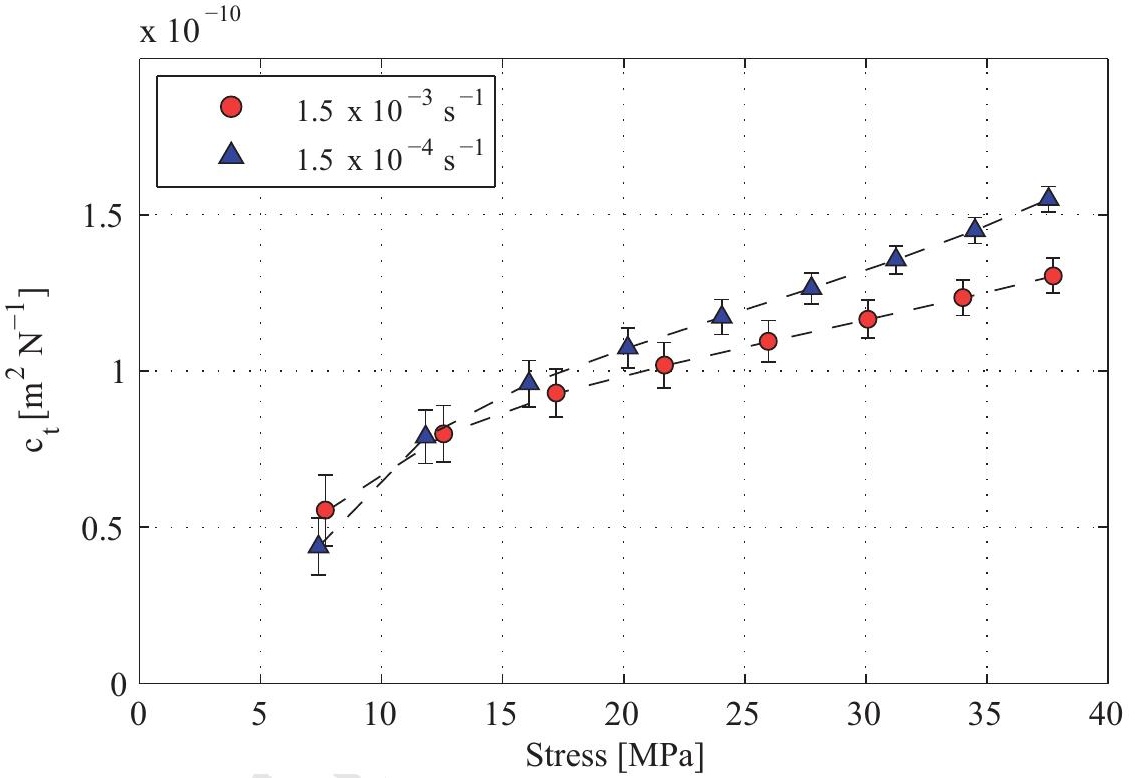




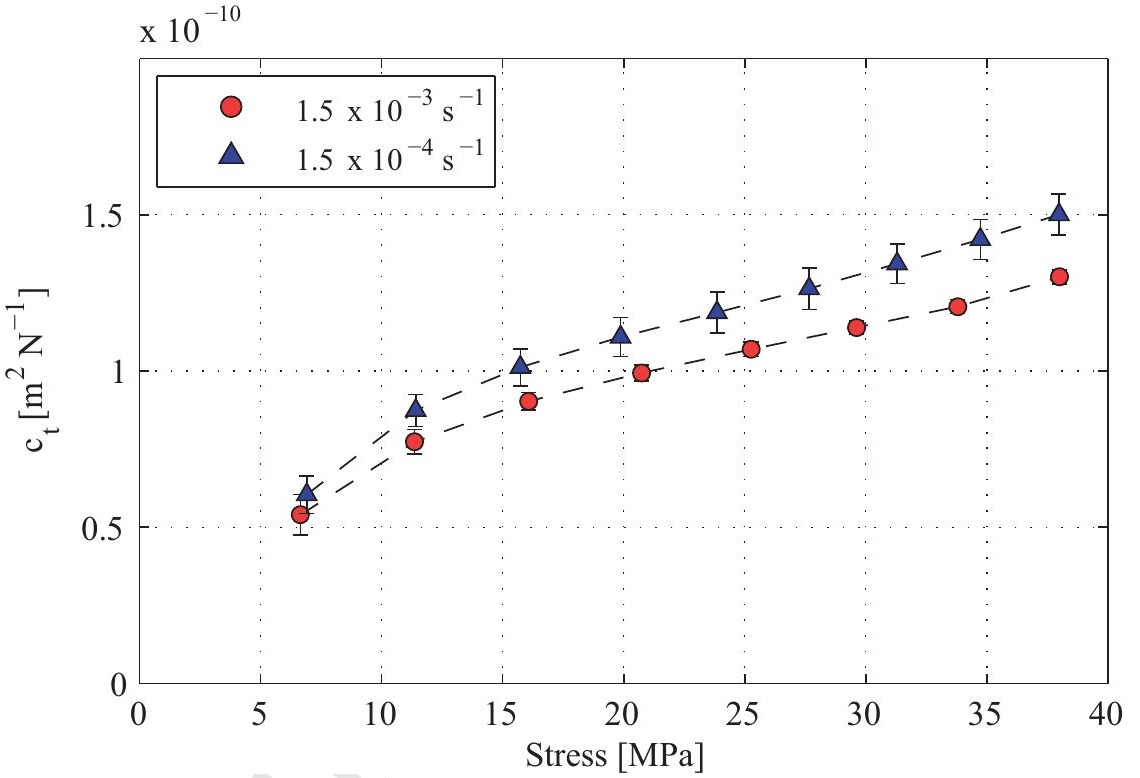




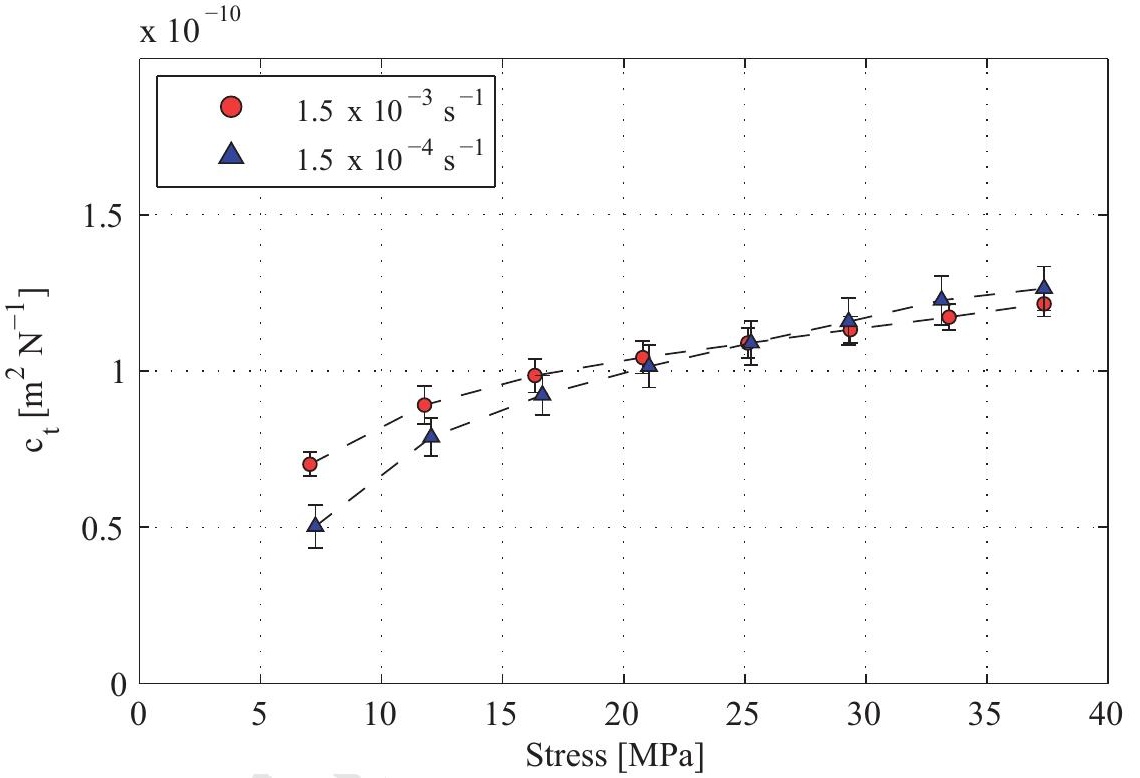




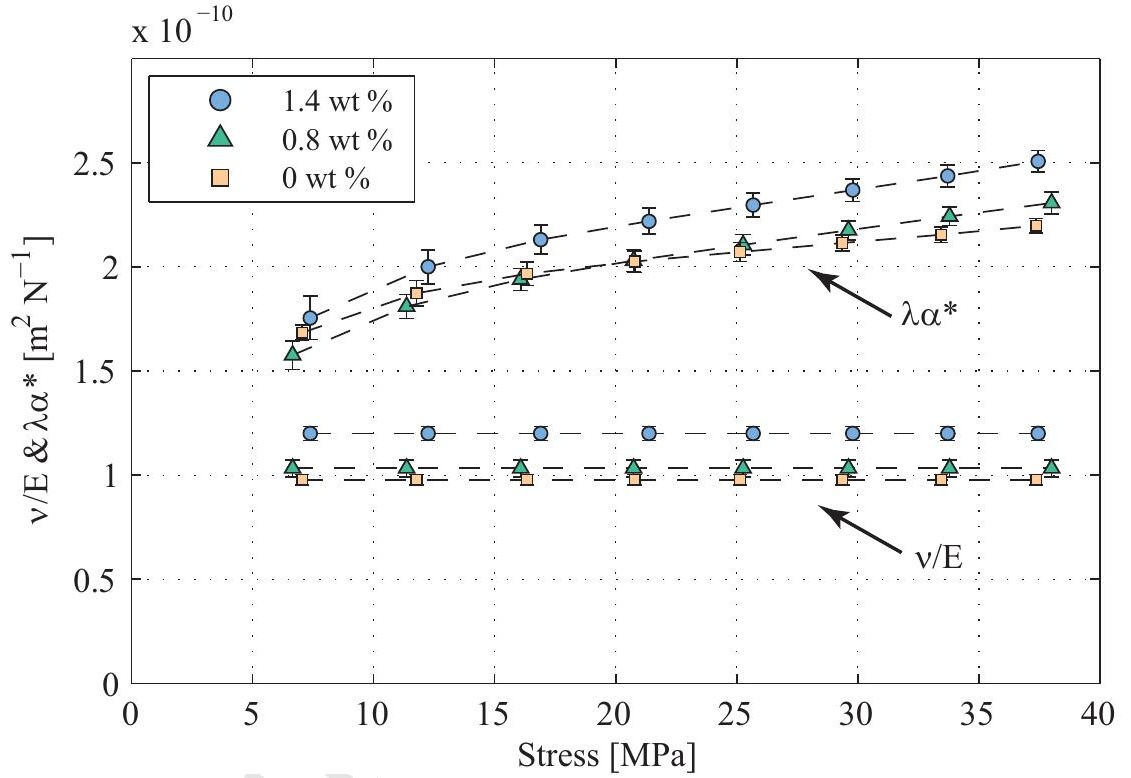




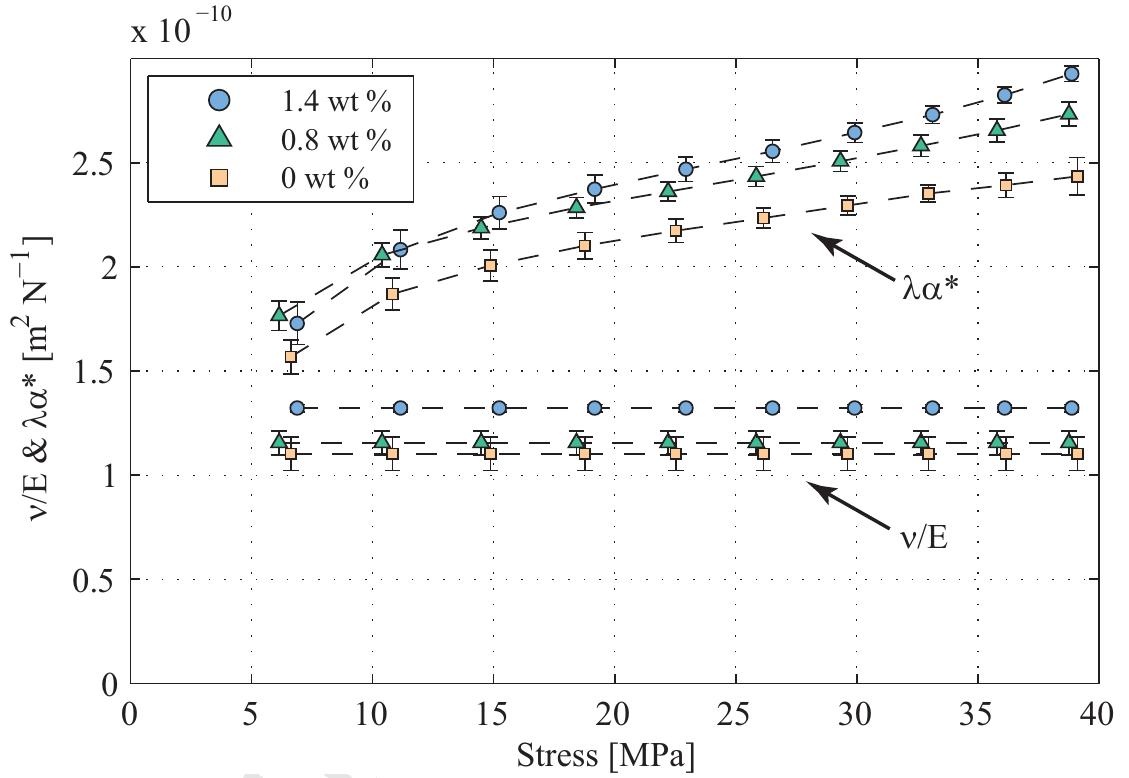

